\title{
From smart working to smart Co-working in the COVID-19 era: a pilot program of cooperation on chronic endocrinological and rheumatological disease
}

\section{Maria Cristina Maggio ( $\square$ mariacristina.maggio@unipa.it)}

University of Palermo https://orcid.org/0000-0002-7777-1239

\section{Claudio Montante}

University Department PROMISE "G. D'Alessandro", University of Palermo

\section{Simona Scalzo}

University Department PROMISE "G. D'Alessandro", University of Palermo

\section{Sofia Felice}

University Department PROMISE "G. D'Alessandro", University of Palermo

Giovanni Corsello

University Department PROMISE "G. D'Alessandro", University of Palermo

\section{Research}

Keywords: telemedicine, paediatrics, chronic disease

Posted Date: October 29th, 2020

DOI: https://doi.org/10.21203/rs.3.rs-97880/v1

License: (c) (1) This work is licensed under a Creative Commons Attribution 4.0 International License.

Read Full License 


\section{Abstract}

Background: The last months were signed by the pandemic diffusion of COVID-19, with the need to minimize the inflow of children and adolescents affected by chronic diseases into the hospitals. Otherwise, paediatricians had to limit visits and to consider a new setting for febrile children.

Patients were assisted by telephonic consultations guaranteed by the paediatricians of free choice and by the paediatric specialists. However, patients frequently needed a direct specialistic evaluation in the case of flares, abnormal laboratory parameters and adverse reactions to drugs.

Another frequent question was the differential diagnosis of febrile episodes, to distinguish a recurrent fever, linked to autoinflammation, from an infectious disease.

We proposed to paediatricians of free choice in west-Sicily a questionnaire about difficulties met in the follow-up of children with rheumatologic diseases, autoinflammatory syndromes, congenital hypothyroidism.

Results: 55 questionnaires were collected: the most frequent recorded conditions were periodic fever, aphthous stomatitis, pharyngitis and cervical adenitis (PFAPA syndrome) and Familial Mediterranean Fever; Juvenile Idiopathic Arthritis, congenital hypothyroidism.

All the paediatricians needed specialistic support to adequately control flares, adjustment of drugs dosage.

Conclusions: Patients and paediatricians need a specialistic support for the follow-up and to reach a good compliance to treatment.

This period characterized by smart working, telemedicine, strategies to monitor remotely the patients, can find the winning strategy in the approach of the "Co-working", a new cooperation between hospital and paediatricians of free choice, in the global follow-up of paediatric chronic diseases.

\section{Background}

Italy's health system has recently been hit by the pandemic diffusion of COVID-19 and, as a matter of fact, access to health care has been limited. Moreover, chronic patients, the most vulnerable especially in pediatric age, risk being neglected or have many difficulties to access to specialistic care (1).

The difficulties emerged after the COVID-19 outbreak, may include: the management of outpatient biochemical and instrumental investigations; the free-access to the tertiary level pediatric care units; the collaboration with specialists (e.g. oculists, orthopedics, dermatologists) allocated in other care units, the frequent access for clinical follow up. In fact, the social distancing does not permit to allow the required number of patients access per day, limiting the possibilities to the direct evaluation by the pediatric specialist. 
These obligations increase the risk of low adherence to treatment and may have serious consequences for the patient as well as for the community health system.

It may also cause a high rate of relapses and required hospitalizations, entailing a negative impact on disease evolution as well as on families quality of life and a social negative impact.

At this stage of emergency, helping people suffering from chronic disease not only means protecting the health of vulnerable subjects, but also protecting public health and health community resources.

Avoiding hospitalization of chronic patients, suffering from relapses in emergency areas because of treatment suspension, not only means protecting patients but also avoiding the spread of the COVID-19 infection.

Therefore, it was necessary to reschedule medical examinations, resulting in postponements and cancellations often requested by parents, to safeguard children suffering from chronic diseases during the outbreak.

Healthcare professionals must be ready to provide appropriate solutions aimed at protecting the wellbeing of patients (2).

This study aims to find solutions shared with pediatricians of free choice (PFC) to properly manage young patients suffering from chronic endocrinological or rheumatological conditions (3).

In this period of public health emergency, connected care solutions, including telemedicine, remote patient monitoring and proper communication between clinicians and patients, may play a pivotal role.

Our aim is to promote a health care system reorganization by technological innovation, while knowing that through the use of technological tools we may encounter a malware at worst!

In the light of our outpatient care-related experience over the last two months, we do believe that reevaluating and improving the relationship between hospital and territory as well as between specialist and PFC can play an instrumental role for a better management of the previous mentioned patients.

In the Smart Working era, we propose a Smart Co-Working program.

\section{Objectives}

- Improving co-management procedures by hospital pediatricians and pediatricians of free choice of patients affected by chronic endocrinological/rheumatological conditions

- Identifying critical points in the management of outpatient examinations in hospital and PFC's clinic

- Assessing the possibility of reducing the hospital inflow by directing it to PFC's clinics

- Identifying new co-management methods with PFC and the patients' families 


\section{Materials And Methods}

The first step of the project was to carry out three surveys, concerning the management of three diseases that often require a specialist pediatrician (e.g. congenital hypothyroidism: $\mathrm{CH}$; autoinflammatory diseases: AID; immuno-rheumatological diseases: IRD).

Assessment questionnaires (see figure 1), administered to all the Sicilian PFCs working in the regions of Palermo, Agrigento, Trapani, Enna, Caltanissetta and Siracusa, were then reviewed by a selected group of PFC who could add any suggestion.

Assessment questionnaires, consisting of 10 questions on average, aimed to evaluate epidemiological data, patients' management and the difficulties encountered by the PFC.

The analysis of these data permitted to customize solutions - primarily based on cooperating with the PFC and supporting patients and their families - aimed to improve the patient management through a chronic care model.

\section{Results}

55 PFC replied to the questionnaires ( $96 \%$ to $\mathrm{CH} ; 85 \%$ to IRD; $100 \%$ to AID, although some of the pediatricians did not reply to all three of the questionnaires we sent.

As we expected, PFAPA syndrome is the most frequent condition observed and followed by PFC in autoinflammatory diseases. The $40.5 \%$ followed the patients with clinical periodic controls and selfmedication by a single dose of betamethasone at the beginning of fever (table 1).

We have evidenced that in most cases colchicine is not employed, whose use

is limited to patients with Familial Mediterranean Fever (FMF) or TRAPS.

Similarly, most of the questions concerning the use of biological drugs and biosimilars showed that a limited number of PFC (17.8\% between PFC following patients with AID; $12.5 \%$ between PFC following patients with IRD) have patients needing these treatments.

According to the PFC, about $50 \%$ of these patients periodically need to go to the hospital for clinical investigations, therapy prescription and/or administration.

These patients do not suffer from PFAPA syndrome that is managed at home by pediatrician.

We have received many feed-back regarding the difficulties in case of flair. In the PFAPA syndrome, autonomy in the management of febrile episodes is generally observed, whereas in other pathologies it is recommended to go to a Reference Specialist Center (figure 1). 
This study underlines some issues concerning family anxiety management resulting from the periodicity of the febrile symptoms.

We have also found that $50 \%$ of PFC have at least one patient suffering from immuno-rheumatological diseases.

Among these diseases, it has been observed the predominance of the oligoarticular JIA, followed by Rheumatic Disease. The last needs periodically the therapeutic plan by the specialist.

The section of the survey concerning therapy showed little feedback on the use of biological drugs. It has also been confirmed that drugs like methotrexate and intra-articular steroids are mostly employed.

52/55 (95\%) of the PFC follows children affected by $\mathrm{CH}$ and such data should be re-evaluated taking into account epidemiology as well as the region in which the pediatrician practices (higher predominance of IC in high endemic areas) (figure 2).

According to the PFC, both the timing in the reevaluation of thyroid function and the remodeling of the therapy with Levothyroxine rely on the specialist competence and require a prompt and specific management in collaboration with a pediatric endocrinologist (figure 3).

In all the questionnaires, colleagues propose a greater cooperation between the specialists and PFC, as well as between the specialist and the patients, through telephone consultations and department email service (designed for both PFC and patients), in order to reduce hospital inflow during the COVID-19 emergency period (figure 4).

It has also been underlined the request of a "technical" discussion about challenging clinical cases between the specialist and the PFC, as well as the importance of realize specialistic training while maintaining social distance (e.g. promoting FAD and Webinars) and studying in-depth these topics, for a better patient co-management.

\section{Discussion}

Because of the COVID-19 emergency, the Italian government had to impose a lockdown to contain the peak of infection. Travelling and moving were permitted only for proven work needs or for documented health reasons.

As a consequence, in Italian hospital units, it was necessary to assess which examinations could be carried out and which could be postponed, according to the urgency of the matter (figure 5).

In this circumstance, the parent of a child with chronic disease, particularly concerned about the health of the child, developed a greater level of anxiety (4).

On the one hand, before a parent reluctant to have his/her son examined by the clinician, we had to explain the urgency and necessity of the examination, on the other hand, some parents, even knowing 
that we only had to perform urgency-related examinations, wanted their child to be examined by the specialist.

According to the guidelines of Italian Society of Pediatrics, patients with chronic diseases, being the most fragile, need an even greater protection (3). In this regard, we do need to develop new management strategies.

Researchers are optimistic about the possibility of obtaining a vaccine against Sars-Cov 2 in the near future. But, before that happens, patients with chronic conditions still remain vulnerable. Furthermore, many of our children receive immunosuppressive therapy and this means that they are more susceptible to infection and that a possible vaccination should be welcome.

Conscious of issues related to our health care-system, we need to readapt to a new society.

The challenge both for patients with chronic diseases and for local health care providers is to reduce travels and limit consultations to specialized centers.

There is also the need to facilitate decentralized care for these patients through a greater cooperation between specialists and local health professionals.

\section{Conclusion}

The Coronavirus emergency highlighted and pushed for an implementation of digital supports for health, to ensure continuity of care and manage chronicity. Thanks to digital health, clinical doctors can be closer to children who are frail and suffer from chronic diseases, like all other patients, while providing home-based services through teleassistance and telemonitoring.

All patients should be introduced as soon as possible to the above-mentioned teleassistance and telemonitoring systems that undoubtedly improve patient adherence to therapies while assuring a better disease management.

The relationship between Specialist and PFC must be the heart of this innovation and the "Smart Working" in healthcare field should become "Smart Co-working", since the management of a pediatric patient suffering from a chronic disease must be based on the cooperation between hospital and territory in order to protect the health of vulnerable children.

\section{Declarations}

Ethics approval and consent to partecipate: not applicable.

Consent for publication: not applicable. 
Availability of data and materials: the datasets used and/or analysed during the current study are available from the corresponding author on reasonable request.

Competing interests: authors have no competing interests to declare.

Funding: authors have no funding to declare.

Authors' contributions: Maria Cristina Maggio: designed the work; Maria Cristina Maggio, Claudio Montante, Simona Scalzo and Sofia Fedele: organized the questionnaires; Maria Cristina Maggio and Giovanni Corsello: revised the paper.

Acknowledgements: the authors acknowledge some paediatricians of free choice, great support in the study: Dr. Antonina Lo Cascio, Dr. Fabio Campo, Dr. Maria Laura La Vecchia and Dr. Maurizio Parisi for the distribution of the questionnairs to paediatricians of free choice in west-Sicily. The authors acknowledge Professor Giuseppe Magazzù (University of Messina) for the useful suggestions during the ideation of the work.

\section{References}

1. Tam LS, Tanaka Y, Handa R, et al. Care for patients with rheumatic diseases during COVID-19 pandemic: A position statement from APLAR [published online ahead of print, 2020 May 27]. Int J Rheum Dis. 2020;10.1111/1756-185X.13863. doi:10.1111/1756-185X.13863c

2. Villani A, Bozzola $E$, Siani P, Corsello $G$. The Italian paediatric society raccomandations on children and adolescents extra-domestic activities during the SARS COV-2 emergency phase 2. Ital J Pediatr. 2020;46(1):62. Published 2020 May 19. doi:10.1186/s13052-020-00826-3

3. Silva CA, Queiroz LB, Fonseca CB, Silva LEVD, Lourenço B, Marques HHS. Spotlight for healthy adolescents and adolescents with preexisting chronic diseases during the COVID-19 pandemic. Clinics (Sao Paulo). 2020;75:e1931. doi:10.6061/clinics/2020/e1931

4. Glasper A. Safeguarding children with long-term conditions from COVID-19. Br J Nurs. 2020;29(9):533-534. doi:10.12968/bjon.2020.29.9.533

\section{Table}

Table 1 is not available with this version

\section{Figures}




\section{Do you think you can handle PFAPA therapy on your own?}

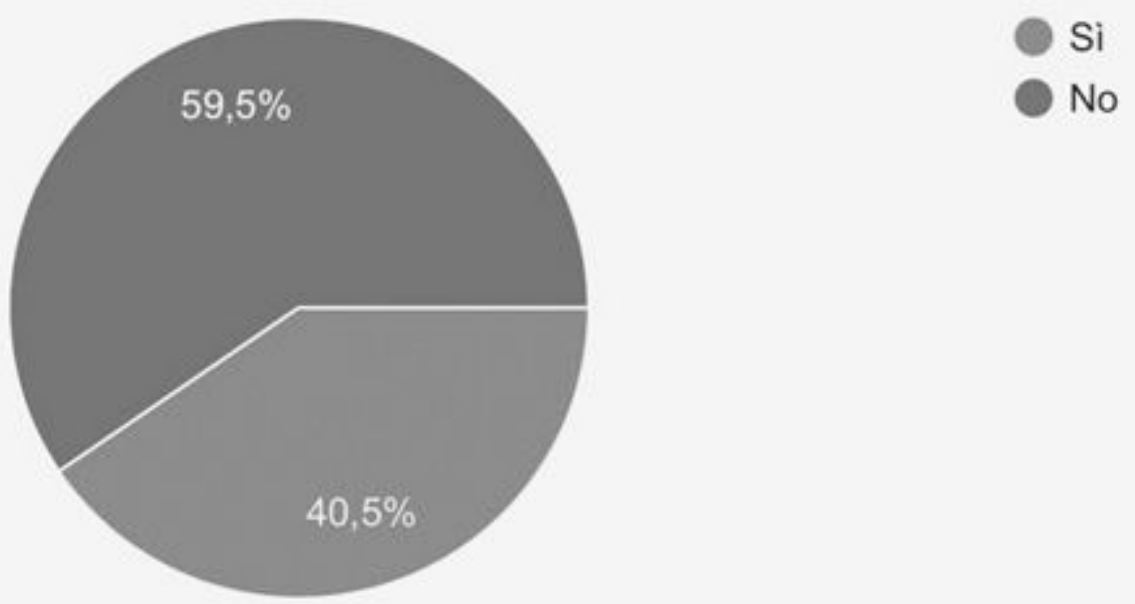

Figure 1

percentage of treatment decision by the specialist and/or by the PFC to treat PFAPA children.

How many patients, with congenital hypothyroidism, do you follow? 15

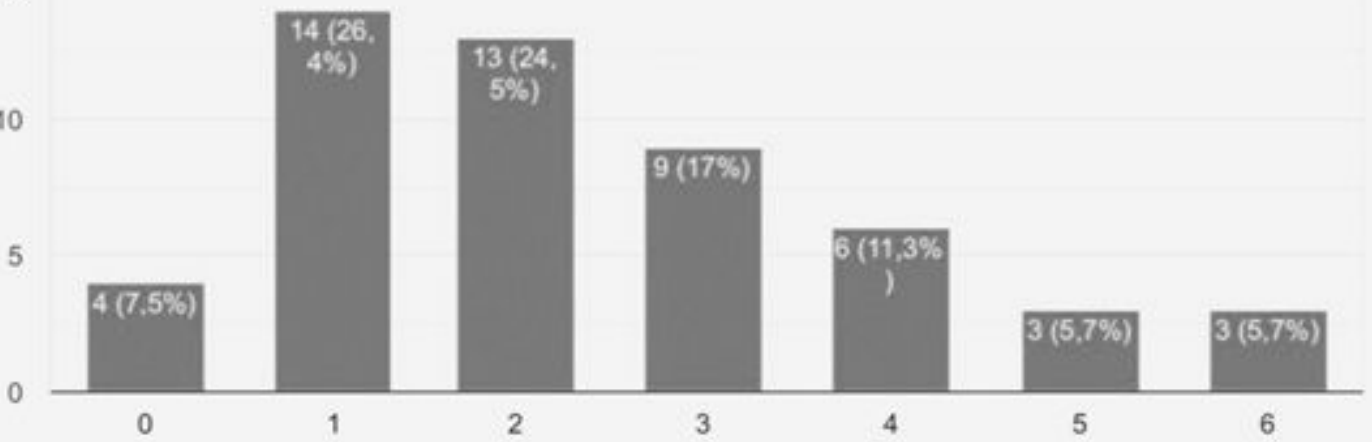

Figure 2

number of $\mathrm{CH}$ patients followed by FCP. 
Who's running the therapy with Levotiroxine?

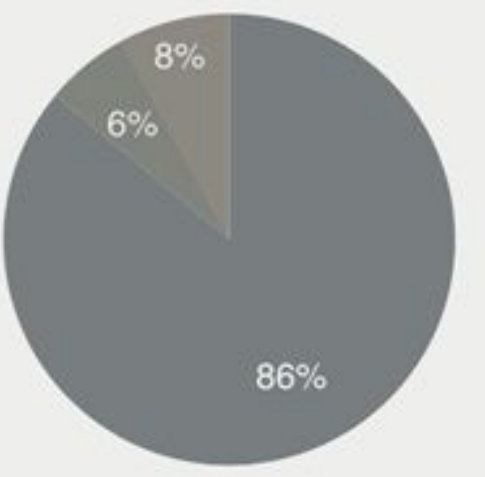

The specialist

PFC

Both

\section{Figure 3}

percentage of treatment decision by the specialist and/or by the PFC.

In relation to the Covid 19 emergency, how do you think the follow-up of these patients could be planned, reducing their access to the hospital?

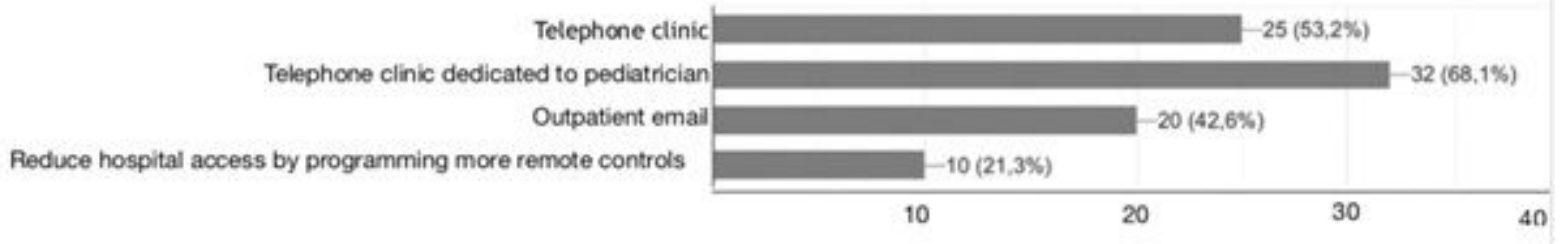

\section{Figure 4}

strategies to reduce patients' access to the hospital.

Who decide the timing of the revalutation thyroid's exams?

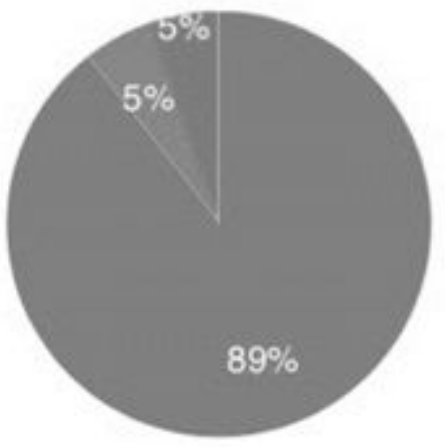

Both

\section{Figure 5}

the decision of the timing to evaluate hormonal parameters in $\mathrm{CH}$ children. 